\title{
The mosquito-handle: Speed and uniformity in de-epithelializing a breast pedicle for reduction mammaplasty
}

S Murphy AFRCSI MD SpR, A Collins AFRCSI MCh SHO, ST O'Sullivan MD FRCS Plast

$\mathrm{D}$

e-epithelialization is a technique practised by many plastic surgeons across a spectrum of procedures. It is commonly used in reduction mammaplasty - a procedure often performed without assistance. De-epithelialization can be laborious; uniformity across the breast pedicle and speed of the procedure is difficult to achieve. The mosquitohandle is a quick, more effective technique of de-epithelialization in reduction mammaplasty.

Reduction mammaplasty can be a laborious and technically demanding procedure, particularly in cases of macromastia or no surgical assistance. The mosquito-handle aids and expedites this procedure.

The breast mound and pedicle is difficult to grasp, and applying uniform tension on both sides of the skin (which is to be removed) is difficult. Uniform tension on the skin is crucial to allow a slick dissection.

Dissection commences around the nipple. A mosquito forceps is used on each side, and the handles are placed opposing one another in the operators' hand. Tension is applied, and dissection continues with the other hand (Figure 1).

This technique is effective, time and energy saving, and cost neutral.

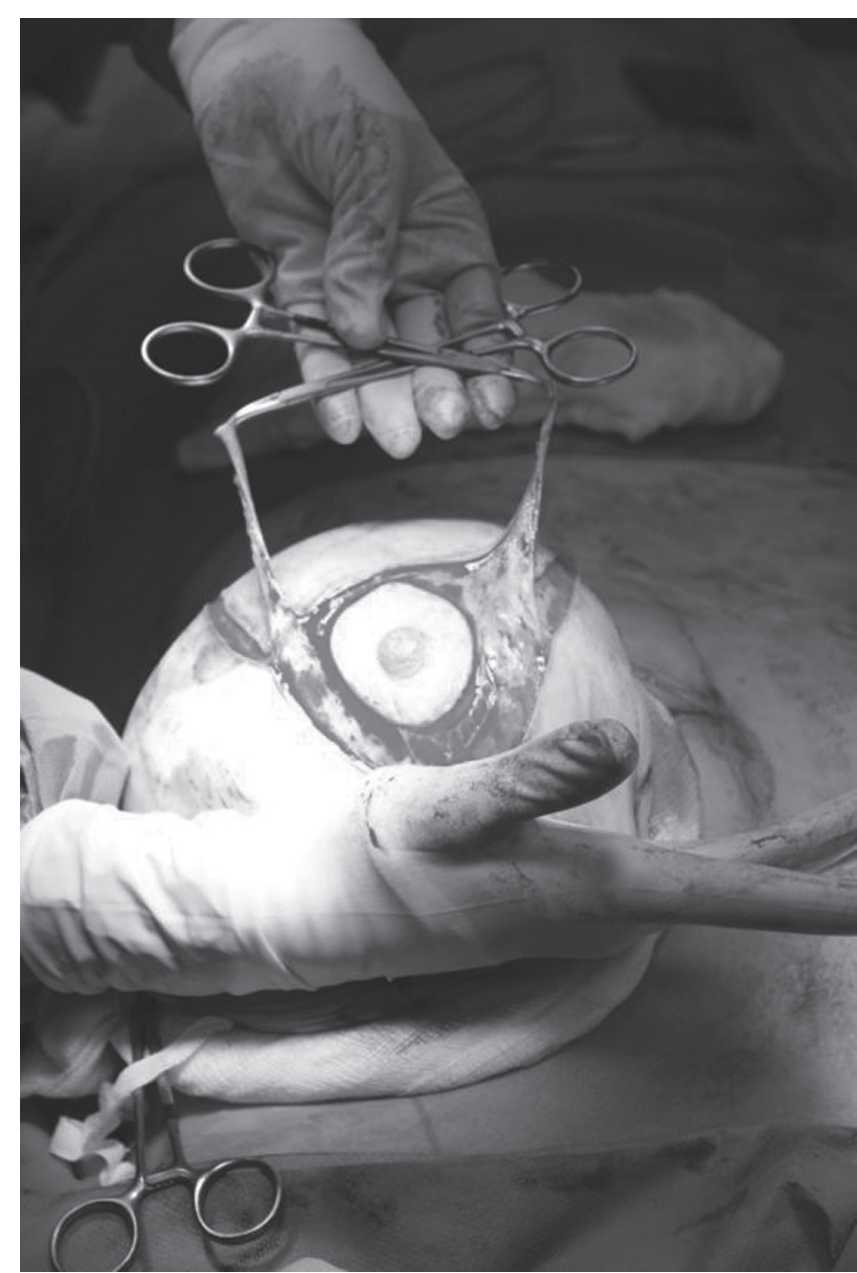

Figure 1) Speed and uniformity of de-epithelializing using the 'mosquito handle' 